\title{
Development of a new HPLC method for wogonin in rat plasma: Compatibility of standard and test samples
}

\author{
JUMAH MASOUD MOHAMMAD SALMANI ${ }^{1}$ \\ XUE WU ${ }^{1}$ \\ JOE ANTONY JACOB \\ RONG FU ${ }^{2}$ \\ BAOAN CHEN ${ }^{*}$ \\ ${ }^{1}$ Department of Hematology \\ and Oncology \\ (Key Department of Jiangsu Medicine) \\ Zhongda Hospital, Medical School \\ Southeast University, Nanjing \\ People's Republic of China \\ 2 State Key Laboratory \\ of Natural Medicines \\ Jiangsu Key Laboratory of Carcinogenesis \\ and Intervention \\ China Pharmaceutical University \\ Nanjing, People's Republic of China
}

Accepted May 24, 2017

Published online June 14, 2017

\begin{abstract}
In the current paper, an HPLC/UV method was developed and validated for determination of wogonin in plasma. Considerable attention was paid to the preparation of standard samples and factors affecting drug distribution. A preparation procedure was devised to simulate the conditions the drug is expected to experience in vivo while pointing to the shortcomings of previously published methods. The method was validated according to the FDA regulations and showed to be highly efficient and capable of extracting the drug and IS from the plasma accurately and precisely within the specified range of $50-500 \mathrm{ng} \mathrm{mL}^{-1}$. Further, the standard sample preparation of this method can be used as a guideline for other methods, particularly when highly hydrophobic drugs with considerable protein binding are involved and could be valuable in the field of bioanalysis to improve the reliability of methods.
\end{abstract}

Keywords: wogonin, HPLC/UV, standard sample preparation, sample compatibility

Knowledge of pharmacologically active species levels in body fluids such as plasma and urine enables researchers to optimize their therapeutic effects (1). Biological fluids and numerous pharmaceutical products are quite complex. Despite the development of highly efficient instruments for analysis, sample preparation (which may take $80 \%$ of analysis time) is still crucial in order to extract and isolate the analytes of interest from such complex media reliably (2). Sample preparation includes test and standard samples where the former requires extraction of a certain analyte from the matrix, while the latter is mainly concerned with spiking specified amounts of the analyte in the matrix of interest, followed by extraction. Compatibility of standard samples is essential because any defects in these samples can affect the reliability of the method; however, this particular issue does not receive adequate attention from researchers and often stays away from the limelight. Standard samples are usually prepared in vitro, which explains the challenges of mimicking the complicated processes that test samples are confronting in vivo. Knowledge about the

*Correspondence; e-mail: cba8888@hotmail.com 
physicochemical properties of the drug must be utilized for optimizing all the phases of bioanalytical methodology, such as spiking, extraction, separation and detection to maximize the chances of effective outcomes (3). During pharmaceutical formulation, some manufacturing processes may change the physical form of the drug. In such cases, it is difficult to validate the accuracy of the method using spiked original analytes, since the recovery of the drug from these standard samples may not represent the recovery from a manufactured product (4). Further, plasma can affect drug distribution in vivo and can even prevent its detection (5). Thus, when the preparation of standard samples does not simulate in vivo conditions, then the recovery of the drug from these standard samples will not represent the recovery from test plasma samples. Method development should consider all the possible affecting variables to achieve optimum yields.

Wogonin, a monoflavonoid extracted from a number of plants, exhibits a wide range of pharmacologic and therapeutic effects, such as antioxidant, anti-inflammatory, neuroprotective, antiviral and antitumor effects (6). Bioanalysis of wogonin is essential for the evaluation of all pharmaceutical preparations intended for clinical use. Most of the previously described methods for the bioanalysis of wogonin were performed using devices such as LC-MS/MS (7-9) or HPLC/UV (10, 11). In contrast, the compatibility of standard samples with test samples was not sufficiently considered in these reports, which is against strict recommendations of the FDA guidelines for the development of bioanalytical methods. In the current investigation, we report a new, simple and robust HPLC/UV method for the determination of wogonin in plasma following FDA regulations and considering variables affecting the standard sample preparation in order to maintain maximum possible compatibility with the test plasma samples.

\section{EXPERIMENTAL}

\section{Preparation of standard stock solutions}

Wogonin standard stock solution was prepared by precisely weighing $5 \mathrm{mg}$ of wogonin, which was first dissolved in $100 \mu \mathrm{L}$ of DMSO (dimethyl sulfoxide), then diluted with methanol to give the final concentration of $50 \mu \mathrm{g} \mathrm{mL}^{-1}$. Then, a set of working standard solutions for spiking standard plasma samples were prepared by dilution with methanol. Apigenin standard stock solution was prepared following the same procedure as for wogonin.

\section{UV-VIS spectrophotometric analysis and HPLC conditions}

Spectrophotometric analysis was performed using an Agilent-8453 (USA) UV-VIS spectrophotometer at $25^{\circ} \mathrm{C}$ to compare the UV/VIS spectra of wogonin and the proposed internal standard solutions (apigenin, IS), and to set an appropriate wavelength for HPLC analysis. An HPLC system (Shimadzu LC-2010C, Japan) equipped with an auto sampler and a diode array detector was used. Chromatographic separation was performed on a Hedera ODS-2 column ( $4.6 \mathrm{~mm}$ in diameter and $250 \mathrm{~mm}$ length, $5 \mu \mathrm{m})$. Isocratic mobile phase was used in this method, as previously reported $(16,22-24)$. It is composed of $75 \%$ of methanol and $25 \%$ of ammonium acetate buffer $\left(77 \mathrm{mg} \mathrm{L}^{-1}, \mathrm{pH}\right.$ adjusted to 4 with acetic acid). Flow rate was $1 \mathrm{~mL} \mathrm{~min}^{-1}$, column temperature was set to $25^{\circ} \mathrm{C}$, injected volume was $30 \mu \mathrm{L}$ and detection wavelength was $277 \mathrm{~nm}$. Chromatographic data was acquired and analysed using the Shimadzu LC solution software package. 
In vitro wogonin aqueous solubility

Briefly, an excess amount of wogonin was kept in PBS (phosphate buffer saline) at $37^{\circ} \mathrm{C}$ in a shaking water bath for 72 hours. The solution was then centrifuged and filtered through a $0.22-\mu \mathrm{m}$ pore filter. Wogonin concentration was then determined by HPLC.

\section{Animal experiments and blood collection}

All animal experiments performed in this study were evaluated and approved by the Animal and Ethics Review Committee of Southeast University (Nanjing, Jiangsu, People's Republic of China). Male Sprague-Dawley rats (body mass around $220 \mathrm{~g}$ ) were monitored twice a day and had free access to regular feed and water. Test animals were deprived of food for at least 12 hours prior to the commencement of the experiment but were freely allowed to drink water. Wogonin aqueous suspension, in a dose of $40 \mathrm{mg} \mathrm{kg}^{-1}$ body mass, was administered orally. Blood collection before and after the treatment was performed through orbital sinus venepuncture utilizing capillary tubes pre-treated with heparin. Around $0.5 \mathrm{~mL}$ of blood was collected each time in small Eppendorf tubes, which were also pretreated with heparin to prevent coagulation. The collected blood was centrifuged for 10 minutes at $10000 \mathrm{rpm}$; plasma was then separated and kept at $-20^{\circ} \mathrm{C}$ until further use.

\section{Optimization of standard sample preparation}

Experiments were performed trying to simulate in vivo conditions. Hence, in order to investigate the effects of spiking organic solvents on the in vivo-in vitro correlation, two groups of standard samples were prepared in duplicate, as illustrated in Fig. 1. For group 1,

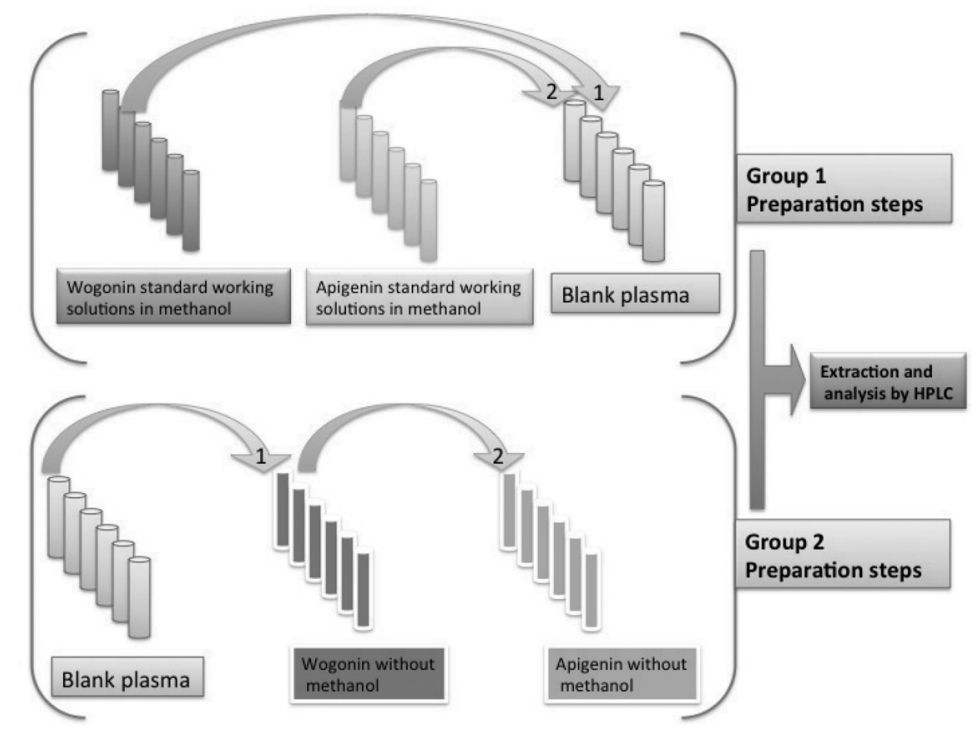

Fig. 1. Schematic presentation illustrating the standard preparation steps in preliminary experiments. 
$10 \mu \mathrm{L}$ of wogonin working solution $\left(0.5,1.25,2.5 \mu \mathrm{g} \mathrm{mL} \mathrm{L}^{-1}\right)$ was directly spiked into $200 \mu \mathrm{L}$ of blank plasma, vortexed and then $10 \mu \mathrm{L}\left(30 \mu \mathrm{g} \mathrm{mL}^{-1}\right)$ of IS stock solution was added. In group 2, $200 \mu \mathrm{L}$ of blank plasma was first added to the same solutions of wogonin but vortexed after evaporating the organic solvent and then moved to tubes that contained the same amount of IS after its organic solvent was evaporated. Both groups, 1 and 2, were vortexed before extraction and analysed by HPLC. Comparison of drug recovery of both sets was used to optimize the standard sample preparation conditions.

\section{Extraction of wogonin and apigenin}

Test plasma samples (from dosed animals) were first added to the IS after evaporation of its organic solvent. The same steps of extraction were performed for both test and standard samples. It started by the addition of ethyl acetate to each sample and vortex for 5 min. Centrifugation was then performed at $12,000 \mathrm{rpm}$ for $5 \mathrm{~min}$ at $4{ }^{\circ} \mathrm{C}$, the clear organic solution was transferred to a clean centrifuge tube and was evaporated to dryness under vacuum. The samples were finally reconstituted with $200 \mu \mathrm{L}$ of methanol and analysed by HPLC after filtration through $0.22-\mu \mathrm{m}$ filters.

\section{Method validation}

In the development phase, experimental conditions of extraction and HPLC procedures (such as the mobile phase components ratio and $\mathrm{pH}$, flow rate and column temperature) were tested to ensure the robustness of the method. The method was then validated according to the FDA guidelines (12).

Calibration curve and linearity. - The calibration curve was developed using a series of blank plasma samples $(200 \mu \mathrm{L})$ spiked with increasing concentrations of wogonin and fixed amounts of IS. The peak area ratio of wogonin to IS was plotted against the concentration to get a standard calibration curve.

Selectivity. - Selectivity of wogonin detection in plasma was tested according to the FDA regulations (12) using drug free blank plasma, blank plasma spiked with wogonin and IS and plasma from wogonin-treated rats spiked with IS. All samples were prepared according to the final optimized conditions and were extracted according to the same method.

Accuracy. - Five standard samples corresponding to each of the low, medium and high concentrations of wogonin were prepared and analysed by HPLC.

Precision. - To ensure precision, five samples corresponding to each of the low, medium and high concentrations of wogonin were prepared according to the optimized method, analysed and the intra-day precision was recorded. Then, each concentration was prepared daily in a sample and analysed for five consecutive days to calculate inter-day precision.

Extraction recovery. - Recovery experiments were performed by comparing the analytical results for extracted standard samples at three concentrations (low, medium, and high) with unextracted standards, representing $100 \%$ recovery. Five samples of each (low, medium and high concentrations of wogonin) were spiked into blank plasma under optimized conditions, extracted and compared with equivalent concentrations of pure wogonin in methanol (without extraction) after HPLC analysis. The mean peak area of the extracted wogonin divided by the mean peak area of the non-extracted wogonin equals the absolute extraction recovery $(n=5)$. 
Limits of detection and quantitation. - Based on the standard deviation of the response and the slope, the limit of detection $(L O D)$ and the limit of quantitation $(L O Q)$ were estimated using the following formula: $L O D=3.3 \sigma / S, L O Q=10 \sigma / S$, where $\sigma$ is standard deviation of the response (standard deviation of y-intercepts of the regression line), $S$ is the slope of the calibration curve.

\section{RESULTS AND DISCUSSION}

\section{Optimization of the method}

Prior to the commencement of HPLC experiments, standard solutions of wogonin and internal standard IS (apigenin) were analysed by UV-VIS spectrophotometry to identify the exact $\lambda_{\text {max }}$. Results (Fig. 2b) showed a prominent peak of wogoninat $277 \mathrm{~nm}$, which is very close to the peak of apigenin (IS) at $268 \mathrm{~nm}$. As a result, $277 \mathrm{~nm}$ was set as the analysis $\lambda_{\max }$.

\section{Selection of the internal standard}

In the current experiment, we selected apigenin (4,5,7-trihydroxyflavone), a flavonoid having a very close structure to wogonin (Fig. 2a). Apigenin shows extremely low aqueous solubility, estimated to be around $5 \mu \mathrm{mol} \mathrm{L}^{-1}$ and $\mathrm{pK} K_{\mathrm{a}}$ value of 7.86 (13). These values are very close to those of wogonin, which has aqueous solubility of $2.34 \mu \mathrm{mol} \mathrm{L}^{-1}(14)$ and $\mathrm{p} K_{\mathrm{a}}$ value of $7.48 \pm 0.1$ (15). The two compounds are therefore expected to have a very low dissociation capacity in aqueous media such as blood plasma. The similarity in these fundamental physicochemical properties makes their recovery from the plasma relatively similar, which makes apigenin a perfect choice for IS in this method. The other two compounds that were primarily considered for IS, since they possess peak UV absorbance very close to that of wogonin, were ibuprofen at $263 \mathrm{~nm}$ and diclofenac sodium at $275 \mathrm{~nm}$. However, they were excluded later not only due to the obvious difference in structure or aqueous solubility but also because, in the current mobile phase, both compounds showed wide peaks that interfered with the peak of wogonin, affecting the method's selectivity.

a)

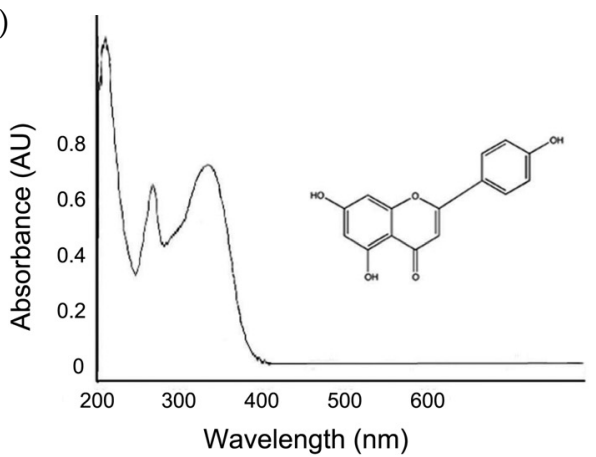

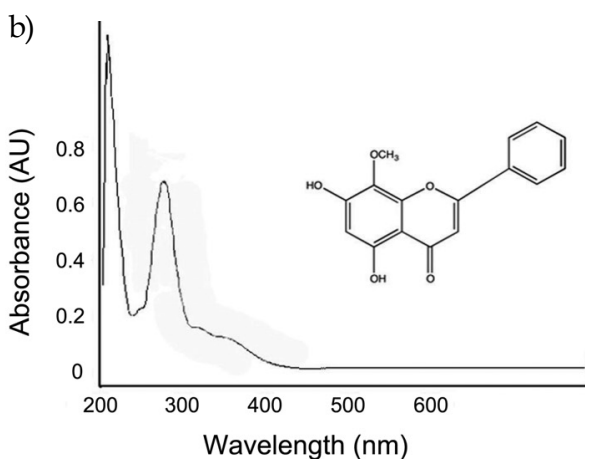

Fig. 2. Molecular structure and UV spectra of: a) apigenin (IS) and b) wogonin. 


\section{Extraction of wogonin and apigenin}

Wogonin and apigenin are expected to be retained in the organic phase (ethyl acetate); the optimized volume ratio ethyl acetate/plasma sample is 5:1. This is to overcome protein binding and recovering the protein bound fraction of the drug, namely, to ensure the quantitative isolation of wogonin and apigenin.

\section{Optimization of standard sample preparation}

Three standard solutions of wogonin with concentrations of $0.5,1.25$ and $2.5 \mu \mathrm{gL}^{-1}$ were primarily used to evaluate the significance of involving an organic solvent in standard sample preparation and its effects on compatibility with the in vivo samples. The results showed recovery of more than $90 \%$ for all concentrations of group 1, in which standard solutions (with organic solvent) were directly spiked into plasma samples. For group 2 , when plasma was added after evaporating the solvent from the standard solution, only the lowest concentration showed high recovery of more than $80 \%$, whereas the other two concentrations exhibited an extreme decline in recovery. As expected, the recovery was inversely proportional to the concentration, reaching only $27 \%$ for the highest concentration of $2.5 \mu \mathrm{gmL}^{-1}$. Therefore, the factors affecting wogonin distribution in plasma were re-assessed and considered to clarify these results and to optimize our method. Among these factors were wogonin aqueous solubility and its protein binding properties, which were thoroughly investigated along with other related factors.

Aqueous solubility of wogonin. - Wogonin solubility in PBS was found to be $2.77 \mu \mathrm{mol} \mathrm{L}^{-1}$, which is very close to the previously reported value for solubility in water of $2.34 \mu \mathrm{mol} \mathrm{L}-1$ (14). It is apparent that wogonin has an extremely low aqueous solubility profile, which can explain the big difference in recovery of the two groups in preliminary experiments. In other words, the addition of drug dissolved in organic solvent disregarded the ability of the plasma to solubilize the drug independently. The drug was solubilized with the aid of the organic solvent and the extraction process cannot represent the extraction from pure plasma, unlike the case in vivo. Consequently, when organic solvents are involved, higher concentrations of wogonin, even more than the capacity of the plasma to solubilize, could be mistakenly used to develop the calibration curve. Hence, such standard samples lack compatibility with the test samples and they may lead to deceptive results and make the method validity questionable. Unfortunately, this was widely done in methods published earlier (10,16-19). Such approaches are in contradiction with the recommendations of the FDA guidance for bioanalytical method validation, which state that the concentrations of standards should be chosen on the basis of the concentration range expected in a particular study (12). Accordingly, we concluded that a maximum concentration of $500 \mathrm{ng} \mathrm{mL}^{-1}$ should be used in the preparation of standard samples to guarantee the ability of plasma to solubilize it freely. Also, the volumes of blank plasma used in standard samples were maintained to independently solubilize the analyte in the concentration range intended for developing the calibration curve.

\section{Wogonin protein binding and plasma distribution}

The calibration curve must be prepared in the same biological matrix as the samples in the planned study by spiking the matrix with known concentrations of the analyte (12). 
Introduction of organic solvents during standard sample preparation will interrupt the composition of the blank plasma matrix, especially if considerable volumes relative to plasma are used. This action must be particularly avoided in order to maintain the compatibility between standard and test samples and the negative consequences for the plasma content and its relation with wogonin distributionare worth mentioning. Organic solvents, particularly methanol, have the ability to precipitate plasma proteins (20). When it is introduced into standard samples, the drug protein-binding pathway will be affected and the drug will be easily recovered (by ethyl acetate) in the extraction process. This can further explain the high recovery of all concentrations of wogonin from group 1 and potentiates our hypothesis of such samples being incompatible with the test samples. Analysis of previous methods of wogonin quantitation in plasma showed that changing the plasma matrix with organic solvents was widely conducted during standard sample preparations $(7,8,10,16-19,21)$. It was performed through direct spiking of the working standard stock solutions of wogonin (with organic solvents) in ratios ranging between 10-50\% of the blank plasma volume while ignoring the consequences for drug solubility and sample integrity and hence their validity. In the current method, in order to prevent such scenarios, the final method protocol was designed to introduce wogonin and IS solely into blank plasma after evaporating the methanol content to preserve its matrix composition and hence compatibility with the test samples.

In the context of protein binding, some drugs and especially those with a high hydrophobic nature have high affinity to binding to plasma proteins by as much as $99 \%$ or higher (5). In addition, covalent protein binding can significantly affect analytical detectability of such pharmaceutical compounds. Although most of drug-protein binding involves non-covalent hydrophobic forces, they are adequate to form a very tight bond to specific sites of some proteins (22). Temperature and time are among the main factors that affect the degree of wogonin protein binding, as previously reported (23-25). These reports showed that the binding process was primarily exothermic, spontaneous, enthalpy and entropy driven. The major part of binding energy was electrostatic interaction accompanied by hydrophobic interaction and hydrogen bonds. In a more recent study, equilibrium dialysis was used to evaluate plasma protein binding of wogonin at different concentrations. The results showed elevated protein binding levels (over $90 \%$ ), which significantly decreased the free fraction of the compound (16). Considering these data, it was concluded that protein binding has a distinctive role in the distribution of wogonin in plasma. Subsequently, in the current procedure of standard sample preparation, specific approaches and techniques were used to optimize the simulation of in vivo conditions. These include the effects of temperature, adequate kinetic energy in addition to evasion of organic solvents that may interfere with protein binding.

Finally, it was obvious that in our preliminary standard sample preparation experiment drug extraction from the directly spiked standard samples (group 1) did not represent real extraction from the plasma matrix and such samples were regarded as incompatible with the test samples. In contrast, in the case when no organic solvent was included in the preparation of standard samples (group 2) only the drug incorporated in plasma was subjected to the extraction process referring to better compatibility with the in vivo test samples. Then the final protocol was established following the general steps in Fig. 1 (Group 2) using a series of $200-\mu \mathrm{L}$ wogonin solutions in methanol (50-500 $\mathrm{ng} \mathrm{mL} \mathrm{m}^{-1}$ ) and a series of $10 \mu \mathrm{L}$ of $30 \mu \mathrm{g} \mathrm{mL} \mathrm{L}^{-1} \mathrm{IS}$ in methanol, all evaporated to dryness. Briefly, plasma was allowed 
to completely thaw at room temperature and then $200 \mu$ Lwas added to each tube of the wogonin series. The tubes were then incubated in a shaking water bath for $20 \mathrm{~min}$ at $37^{\circ} \mathrm{C}$ interspersed with 1-min vortex periods every $5 \mathrm{~min}$ to provide thermal conditions and sufficient mixing required to incorporate the drug under simulated in vivo conditions. It was then completely transferred to the series that contained IS and vortexed for $5 \mathrm{~min}$. Addition of IS was carried out in a separate step in order to avoid any possible interference with the drug distribution pattern such as competition on protein binding sites or, more importantly, to mimic the addition sequence of IS to the test samples and hence ensure higher compatibility from all aspects. This was followed by the extraction process (according to the same protocol as with test samples) and then analysing by HPLC.

\section{Method validation}

Linearity of calibration curve. - Standard samples prepared according to the final protocol were extracted and analysed by HPLC and were repeated to fulfil the requirement recommended by the FDA bioanalytical guideline for method validation. Coefficient of determination $R^{2}$ of 0.99899 was valid throughout the linearity concentration range of 50-500 ng mL $\mathrm{mL}^{-1}$, appropriate for studying wogonin pharmacokinetic behaviour in vivo. It is the only range in which the result of the method is trustworthy and, in addition, this range reflects the real concentrations of the drug in aqueous solutions in general or plasma in particular.

Selectivity. - The peak of IS and wogonin eluted at 5.1 and 7.2 min, resp., with relative retention time RRT of 1.4. HPLC chromatograms for blank plasma, blank plasma spiked with wogonin and IS and test plasma from wogonin-treated rat spiked with IS are shown in Figs. 3a,b,c. Apparently, no interference was found at retention times of IS and wogonin, indicating the selectivity of the method for their separation from both standard and test samples, pointing to the applicability of the method for the pharmacokinetic studies of wogonin in vivo.

Accuracy. - The method was shown to be accurate since the mean values of the three concentrations were very close to their corresponding true values, specifically satisfying the FDA recommendation of being within $15 \%$ of the true value (12). Table I shows the intra- and inter-day accuracy and RSD values for each mean value of the three concentrations tested.

Precision. - Precision of the method was confirmed by the results of intra-day repeatability and inter-day (intermediate) precision, which showed the closeness of individual measures of each concentration when the procedure was applied repeatedly. Maximum RSD values of 11 and $2 \%$ for the lowest and the highest concentration, resp., which are within the allowed limits of the FDA regulations (15\% RSD) (12), were recorded when the experiment was repeated on different days (Table I).

Extraction recovery. - The recorded average absolute recovery value of the current method was about $85 \%$ (Table II). These results confirm the method applicability and validity for the determination of wogonin from plasma within the specified range.

Limit of detection and quantitation. - The method was proven to be extremely sensitive, with $L O D$ of $10.5 \mathrm{ng} \mathrm{mL}^{-1}$ and $L O Q$ of $31.7 \mathrm{ng} \mathrm{mL}^{-1}$, suggesting its suitability for bioavailability studies of oral and parenteral administration of wogonin. 
a)

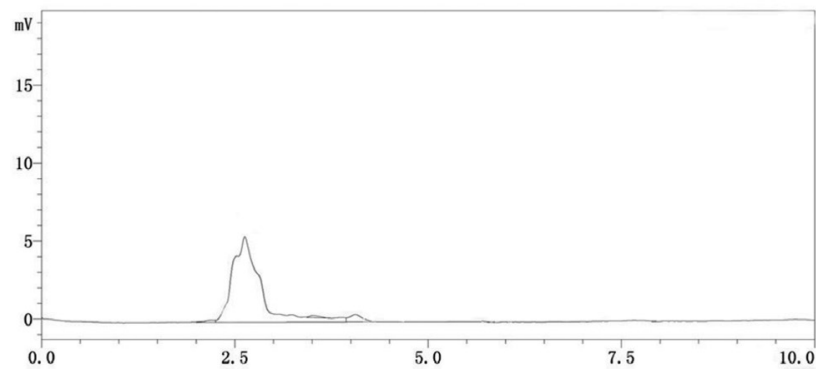

b)

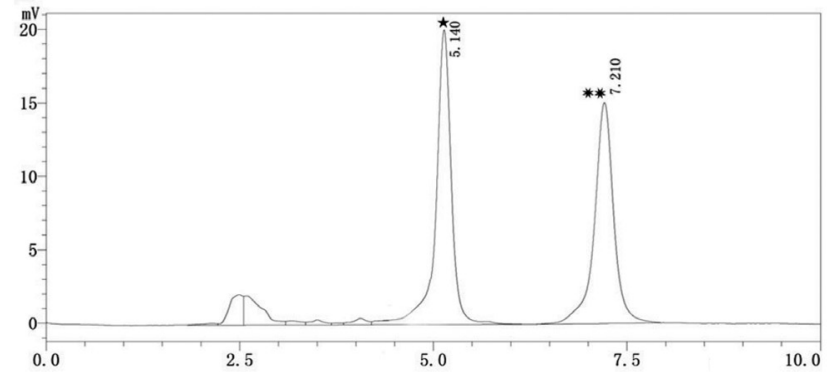

c)

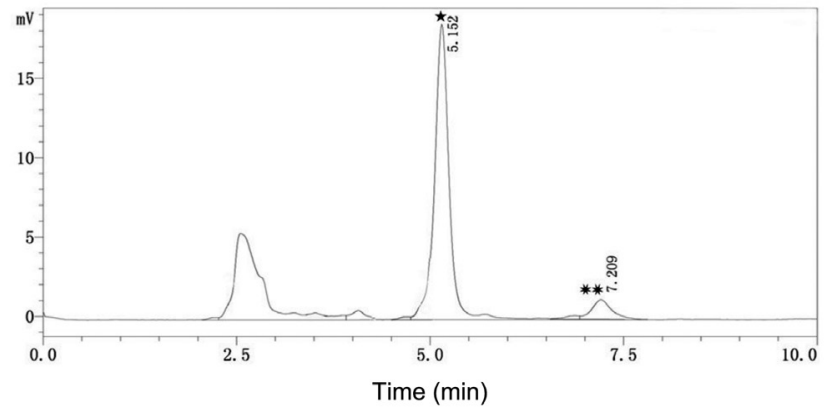

Fig. 3. HPLC chromatograms of: a) blank plasma, b) blank plasma spiked with wogonin and apigenin, c) plasma sample from a rat treated with wogonin aqueous suspension per os. Key to the peaks: ${ }^{*}$ apigenin, ${ }^{* *}$ wogonin.

Table I. Method accuracy and precision

\begin{tabular}{ccccccc}
\hline & Inter-day & \multicolumn{3}{c}{ Intra-day } \\
\hline $\begin{array}{c}\text { True wogonin } \\
\text { conc. }\left(\text { ng mL }^{-1} \text { ) }\right.\end{array}$ & $\begin{array}{c}\text { Measured } \\
\text { wogonin conc. } \\
\left(\mathrm{ng} \mathrm{mL}^{-1}\right)\end{array}$ & $\begin{array}{c}\text { RSD } \\
(\%)\end{array}$ & $\begin{array}{c}\text { Accuracy } \\
(\%)\end{array}$ & $\begin{array}{c}\text { Measured } \\
\text { wogonin } \\
\text { conc. }\left(\mathrm{ng} \mathrm{mL} \mathrm{mL}^{-1}\right)\end{array}$ & $\begin{array}{c}\text { RSD } \\
(\%)\end{array}$ & $\begin{array}{c}\text { Accuracy } \\
(\%)\end{array}$ \\
\hline 50.00 & 46.60 & 11.0 & 93.2 & 45.25 & 9.5 & 90.5 \\
300.00 & 285.57 & 2.9 & 95.2 & 291.34 & 1.3 & 97.1 \\
500.00 & 488.42 & 2.0 & 97.7 & 484.62 & 1.4 & 96.9 \\
\hline
\end{tabular}

$n=5$ 
Table II. Method recovery

\begin{tabular}{ccc}
\hline True wogonin conc. $\left(\mathrm{ng} \mathrm{mL}^{-1}\right)$ & Recovery $(\%)$ & RSD $(\%)$ \\
\hline 50.00 & 85.4 & 7.3 \\
300.00 & 87.3 & 7.5 \\
500.00 & 83.2 & 5.5 \\
\hline
\end{tabular}

$n=5$

\section{CONCLUSIONS}

The current work objectifies the weak points of previous preparations of standard samples such as spiking organic solvents in the plasma, illogical calibration concentration range and disregard of the effects of physicochemical properties of the drug and experimental conditions. The current method also suggests applicable measures for overcoming these drawbacks.

Standard sample preparation during the development of an HPLC method for wogonin determination in rat plasma was discussed in detail, pointing to the significance of incompatibility between standard and test samples. It showed that method validation does not fulfil its purpose and no reliable results will be achieved if wrong standards are used. Further, current experiments were preformed considering all factors necessary to make the results more valuable and trustworthy. This research highlights the importance of proper execution of the standard sample preparation, particularly for analytes with low aqueous solubility and high protein binding. To the best of our knowledge, this is the first report emphasizing the phenomena of inappropriate implementation of standard guidelines in standard sample preparation that affect their compatibility and hence the entire method reliability. Due to the great importance of preventing deceptive results obtained if the method is validated using incompatible standard samples, this research aims to correct such drawbacks by designating the measures for achieving the required compatibility.

Further, this method was validated and was shown to be highly efficient and capable of extracting wogonin and IS (apigenin) from rat plasma accurately and precisely within the specified range, thus suggesting its suitability for wogonin determination in plasma.

Since the incompatibility issue has not yet been considered as the primary objective of method development, this report could be valuable in the bioanalysis of other organic compounds to avoid such problems in this early and essential step of method development.

Acknowledgments. - This work was supported by the key medical projects of Jiangsu province (No.: BL2014078), the key department of Jiangsu medicine (No.: 2012-12), National Nature Science Foundation of China (No.: 81170492), the National Nature Science of China (No.: 81370673), Jiangsu Province Health Department Medical Science and Technology Development Fund Project (No.: H201229), and the Key Department of Jiangsu Medicine (No.: 2011-12).

\section{REFERENCES}

1. H. Kataoka and H. L. Lord, Sampling and Sample Preparation for Clinical and Pharmaceutical Analysis, in Sample and Sample Preparation for Field and Laboratory (Ed. J. Pawliszyn), $1^{\text {st }}$ ed., Elsevier, Amsterdam 2002. 
2. F. Xiaofang, L. Yiping and L.Huwei, Sample preparation for pharmaceutical analysis, 381 (2005) 75-77; DOI: 10.1007/s00216-004-2894-5.

3. R. F Venn, Physicochemical Properties of Drugs and Metabolites and their Impact on Bioanalysis, in Principles and Practice of Bioanalysis, $2^{\text {nd }}$ ed., CRC Press, New York 2008.

4. L. Ohannesian and A. Streeter, Handbook of Pharmaceutical Analysis, Taylor \& Francis, New York 2001.

5. N. El-Najjar, R. A. Ketola, T. Nissila, T. Mauriala, M. Antopolsky, J. Janis, H. Gali-Muhtasib, A. Urtti and $\mathrm{H}$. Vuorela, Impact of protein binding on the analytical detectability and anticancer activity of thymoquinone, J. Chem. Biol. 4 (2011) 97-107; DOI: 10.1007/s12154-010-0052-4.

6. T. Li, N. Li, Q. Guo, H. Ji, D. Zhao, S. Xie, X. Li, Z. Qiu, D. Han, X. Chen and Q. You, Inhibitory effects of wogonin on catalytic activity of cytochrome P450 enzyme in human liver microsomes, Eur. J. Drug Metab. Pharmacokinet. 36 (2011) 249-256; DOI: 10.1007/s13318-011-0050-0.

7. L. Jian-chun, C. Fei-hu, D. Hai-jun and G. Shu, Study on plasma concentration and bioavailability of wogonin in beagle dogs, Chin. Herbal Med. 3 (2011) 144-149; DOI: 10.3969/j.issn.16746384.2011.02.010.

8. Y. H. Kim, D. W. Jeong, I. B. Paek, H. Y. Ji, Y. C. Kim, D. H. Sohn and H. S. Lee, Liquid chromatography with tandem mass spectrometry for the simultaneous determination of baicalein, baicalin, oroxylin A and wogonin in rat plasma, J. Chromatogr. B 844 (2006) 261-267; DOI: 10.1016/j. jchromb.2006.07.021.

9. Y. H. Kim, D. W. Jeong, Y. C. Kim, D. H. Sohn, E. S. Park and H. S. Lee, Pharmacokinetics of baicalein, baicalin and wogonin after oral administration of a standardized extract of Scutellaria baicalensis, PF-2405 in rats, Arch. Pharm. Res. 30 (2007) 260-265.

10. C. Li, L. Zhang, G. Lin and Z. Zuo, Identification and quantification of baicalein, wogonin, oroxylin A and their major glucuronide conjugated metabolites in rat plasma after oral administration of Radix scutellariae product, J. Pharm. Biomed. Anal. 54 (2011) 750-758; DOI: 10.1016/j.jpba.2010.10.005.

11. T. H. Tsai, C. J. Chou, T. R. Tsai and C. F. Chen, Determination of wogonin in rat plasma by liquid chromatography and its pharmacokinetic application, Planta Med. 62 (1996) 263-266; DOI: 10.1055/s2006-957874.

12. FDA (CDER and CVM), Guidance for Industry - Bioanalytical Method Validation, U.S. Department of Health and Human Services - Food and Drug Administration, Rockville (MD) May 2001.

13. Z. E. Pápay, Z. Sebestyén, K. Ludányi, N. Kállai, E. Balogh, A. Kósa, S. Somavarapu, B. Böddi and I. Antal, Comparative evaluation of the effect of cyclodextrins and $\mathrm{pH}$ on aqueous solubility of apigenin, J. Pharm. Biomed. Anal. 117 (2016) 210-216; DOI: 10.1016/j.jpba.2015.08.019.

14. J. Sun, F. Li, Y. Zhao, L. Zhao, C. Qiao, Z. Li, Q. Guo and N. Lu, LZ-207, a newly synthesized flavonoid, induces apoptosis and suppresses inflammation-related colon cancer by inhibiting the NFкB signaling pathway, PLoS ONE10 (2015) e0127282; DOI: 10.1371/journal.pone.0127282.

15. L. Tingting, K. Guo-jun, H. Hui-ling and Z. Lei, Determination of $\mathrm{p} K_{\mathrm{a}}$ values of wogonoside, wogonin and oroxylin-A by RP-HPLC, Chin. J. Pharm. Anal. 8 (2014) 1475-1480.

16. A. Talbi, D. Zhao, Q. Liu, J. Li, A. Fan, W. Yang, X. Han and X. Chen, Pharmacokinetics, tissue distribution, excretion and plasma protein binding studies of wogonin in rats, Molecules 19 (2014) 5538-5549; DOI: 10.3390/molecules19055538.

17. C. Xiaoyan, W. Hongyan, D. Yue and Z. Dafang, Quantitation of the flavonoid wogonin and its major metabolite wogonin-7 $\beta$-d-glucuronide in rat plasma by liquid chromatography-tandem mass spectrometry, J. Chromatogr. B 775 (2002) 169-178; DOI: 10.1016/S1570-0232(02)00288-X.

18. L. Wei, Comparison of the tissue distributions of flavonoids after oral administration of pure baicalin, Radix scutellariae and Scutellariae-Paeoniae couple extracts to rats, J. Med. Plants Res. 5 (2011) 6907-6915; DOI: 10.5897/jmpr11.410. 
19. L. Tong, M. Wan, L. Zhang, Y. Zhu, H. Sun and K. Bi, Simultaneous determination of baicalin, wogonoside, baicalein, wogonin, oroxylin A and chrysin of Radix scutellariae extract in rat plasma by liquid chromatography tandem mass spectrometry, J. Pharm. Biomed. Anal. 70 (2012) 6-12; DOI: 10.1016/j.jpba.2012.03.051.

20. S. N. L. Grace and J. A. J. H. Critchley, The estimation of paracetamol and its major metabolites in both plasma and urine by a single high-performance liquid chromatography assay, J. Pharm. Biomed. Anal. 12 (1994) 1563-1572; DOI: 10.1016/0731-7085(94)00859-0.

21. S. Y. Fong, Y. C. Wong and Z. Zuo, Development of a SPE-LC/MS/MS method for simultaneous quantification of baicalein, wogonin, oroxylin $A$ and their glucuronides baicalin, wogonoside and oroxyloside in rats and its application to brain uptake and plasma pharmacokinetic studies, J. Pharm. Biomed. Anal. 97 (2014) 9-23; DOI: 10.1016/j.jpba.2014.03.033.

22. K. Maruyama, H. Nishigor and M. Iwatsuru, Location of drug binding sites on human serum albumin, Chem. Pharm. Bull. 34 (1986) 2989-2993.

23. Y. Liu, W. He, W. Gao, Z. Hu and X. Chen, Binding of wogonin to human gamma globulin, Int. J. Biol. Macromol. 37 (2005) 1-11; DOI: 10.1016/j.ijbiomac.2005.04.005.

24. J. Tian, J. Liu, J. Xie, X. Yao, Z. Hu and X. Chen, Binding of wogonin to human serum albumin: a common binding site of wogonin in subdomain IIA, J. Photochem. Photobiol. B 74 (2004) 39-45; DOI: 10.1016/j.jphotobiol.2004.01.001.

25. J. Tian, J. Liu, Z. Hu and X. Chen, Interaction of wogonin with bovine serum albumin, Bioorg. Med. Chem. 13 (2005) 4124-4129; DOI: 10.1016/j.bmc.2005.02.065. 